Int. J. Dev. Biol. 53: 1023-1033 (2009)

doi: $10.1387 / \mathrm{ijdb} .082780 \mathrm{vt}$

\title{
The surface ectoderm of the chick embryo exhibits dynamic variation in its response to neurogenic signals
}

\author{
VINEETA B. TRIPATHI*,\#, YASUO ISHI\#\#, MUHAMMAD M. ABU-ELMAGD\#\#\# \\ and PAUL J. SCOTTING \\ Children's Brain Tumour Research Centre, Institute of Genetics, University of Nottingham, Queen's Medical Centre, Nottingham, UK
}

\begin{abstract}
The epibranchial placodes are specialized areas of surface ectoderm that make a vital contribution to the peripheral nervous system, producing sensory neurons of the cranial ganglia. They have long been characterized as a series of patches of thickened ectoderm in the vicinity of each pharyngeal cleft. We have previously demonstrated that Sox 3 is not only expressed in these structures but also marks a larger, earlier domain. Here we demonstrate that neurons are produced from the Sox3-positive ectoderm that lies outside of the classically-defined epibranchial placodes. Our data show that these regions contribute neurons to the cranial ganglia, but then cease producing neurons as they lose Sox3 expression. We further demonstrate that the ectoderm in these regions is responsive to extracellular or intracellular stimuli that initiate aspects of neuronal differentiation. This response to neurogenic stimuli is lacking in regions of ectoderm distant from the normal sites of neurogenesis and the response to constitutively active Bmp receptor in particular, disappears coincident with loss of Sox3 expression. Finally, we show that a dominant repressor form of Sox3 blocks the ability of the ectoderm to undergo neurogenesis. Thus, Sox 3 appears to be essential for the neurogenic capacity of surface ectoderm exhibited by the epibranchial placodes.
\end{abstract}

KEY WORDS: placode, Sox3, neurogenin, neuroD, Bmp, chick

\section{Introduction}

In vertebrates, the regions of origin of the nervous system can be divided into three components. The central nervous system (brain and spinal cord) arises from the neural plate, while the peripheral nervous system arises from the neural crest and the neurogenic placodes. Despite the different origins of these components, they share many features in common (Streit, 2004, Schlosser, 2005). They are usually comprised of thickened columnar, pseudostratified epithelium that can be easily distinguished from the surrounding epidermal cells. Upon neurogenesis these cells undergo transition from an epithelial to a migratory state and express many genes in a similar sequential order.

Our current models of neuronal development primarily depend upon our knowledge of the central nervous system. Neuronal development in the central nervous system is a complex process involving many steps and signaling events (Bally-Cuif and Hammerschmidt, 2003). The earliest steps of this process appear to involve epithelial cells of the early embryo acquiring the capacity to become neural precursors. These 'competent' precursors subsequently adopt a neural fate in response to neural inducing signals leading to the formation of the neural plate. Neurogenesis takes place only later and is spatially and temporally regulated by complex regional and cell-type specification events.

The neurons of the distal ganglia of cranial nerves VII, IX and $X$ (geniculate, petrosal and nodose ganglia) arise from the

Abbreviations used in this paper: BMP, bone morphogenetic protein.

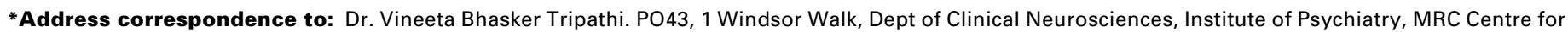
Neurodegeneration Research, King's College London, Denmark Hill, London SE5 8AF, UK. Fax: +44-20-7848-0988. e-mail: Vineeta.Tripathi@iop.kcl.ac.uk

Supplementary Material for this paper, consisting of one figure is available at: http://dx.doi.org/10.1387/ijdb.082780vt

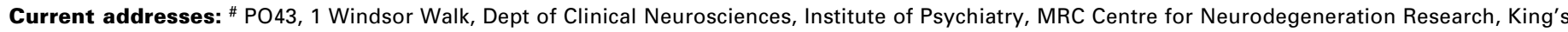

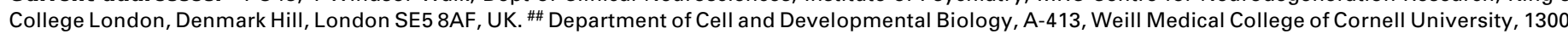
York Avenue, New York, NY 10021, USA. \#\#\# School of Biological Sciences, The University of East Anglia, Norwich, NR4 7TJ, UK. 
epibranchial placodes in the vicinity of the pharyngeal clefts (Le Douarin et al., 1986, Webb and Noden, 1993, Graham and Begbie, 2000, Baker and Bronner-Fraser, 2001). Because of their location and simplicity, these placodes provide an attractive system to understand fundamental mechanisms underlying neural fate. They are classified into a ventrolateral series that produce only sensory neurons; all the non-neuronal components of the ganglia are of neural crest origin (Narayanan and Narayanan, 1980, D’Amico-Martel and Noden, 1983).

Despite their importance to the cranial nervous system, little is known about formation and early development of the epibranchial placodes. Although many molecular markers for placodal neurogenesis have recently been identified (Baker and BronnerFraser, 2001) some basic features of the placodes still remain unclear. We have shown that Sox3, an HMG family transcription factor, is expressed in the classically defined epibranchial placodes, which are located dorsocaudal to each pharyngeal cleft (AbuElmagd et al., 2001, Ishii et al., 2001). However, Sox3also marks a larger domain in the early post-otic region, within which three of the epibranchial placodes arise through loss of Sox3expression in the intervening ectoderm (Ishii et al., 2001).

We have set out to determine whether the dynamically changing domains of Sox3 expression reflect changes in the ability of ectoderm to undergo neurogenesis. We first determined when and where neurogenesis occurred in relation to Sox3expression using in situ hybridization and fate mapping. We then analysed temporal and spatial variation in the response of ectoderm to extracellular (activated BMP receptor) or intracellular (neurogenin 2) stimuli of neurogenesis. Finally, we determined whether Sox3 function was necessary for the competence to undergo neurogenesis. These analyses reveal that the early domains of Sox3 expression are indeed neurogenic and that Sox3 is necessary for the cells of the placodes to undergo neurogenesis.

\section{Results}

\section{The relationship between Sox3-expressing ectoderm and expression of the neurogenic $\mathrm{bHLH}$ factors}

We have previously shown that Sox3 is expressed in the epibranchial placodes of developing chick embryos (Abu-Elmagd et al., 2001, Ishii et al., 2001), also see Fig. 1E). However, our studies also showed that these final patches of Sox3 expression were the remnants of fewer, much larger domains of expression. This means that a large part of the early domains of Sox3 expression include regions of ectoderm in which neurogenesis has not been described. In order to gain further insight into the relationship between Sox3 expression and neurogenesis, we examined the appearance of the bHLH gene transcripts Neurogenin 1 and NeuroD, during the period when the domains of Sox3 expression were shrinking and segmenting. By stage 13, Sox3 was expressed in a broad domain (Fig. $1 \mathrm{~A})$, which contains all prospective regions of the final epibranchial placodes except the pre-otic geniculate placode (Ishii et al., 2001). By stage 14, Sox3 expression started to be lost from the ectoderm fated to lie between the petrosal and nodose placodes (Fig. 1B). The level of Sox3 expression in this ectoderm declined gradually in a dorsal to ventral fashion (Fig. $1 B-D)$, and consequently the petrosal and nodose placodes appeared by stage 17 as two distinct patches of Sox3 expres- sion (Fig. 1D). The nodose placode was further resolved into two by stage 19 (Fig. 1E), again through the loss of Sox3 expression in the intervening region (Ishii et al., 2001). At each stage the regions of Sox3 expression were coincident with the thickened 'placodal' ectoderm (Supplementary Fig 1).

Neurogenin 1, the earliest known gene to be expressed when a placodal cell starts neuronal differentiation (Abu-Elmagd et al., 2001) has been suggested to act as a neuronal determination factor through regulating many downstream genes, including NeuroD (Fode et al., 1998, Perez et al., 1999). Neurogenin 1 and NeuroD started to be expressed by stage 13 and 14 , respectively, in a small number of scattered cells in and around the prospective region of the petrosal placode (Fig. 1F$\mathrm{H}, 1 \mathrm{~K}-\mathrm{M})$. Double in situ hybridization for NeuroD and Sox3 demonstrated that the NeuroD-positive neuroblasts delaminate from within the Sox3-positive ectoderm (Fig. 1P-T). After stage 16, the number of cells expressing Neurogenin 1 and NeuroD increased and became restricted to the classically defined petrosal and nodose placodes (Fig. 1I, 1J, 1N and 10). This restriction appeared to be concomitant with loss of Sox3 expression from the intervening ectoderm.

\section{Regions of neurogenesis change with time}

Our previous fate mapping study demonstrated that the petrosal and nodose placodes arise from two separate areas of the pharyngeal ectoderm at stage 13 (Ishii et al., 2001). However, the expression of bHLH transcription factors described above suggests that placodal neurogenesis is continuous across this region even at stage 16 (Fig. $1 \mathrm{H}$ and $1 \mathrm{M})$. It is likely therefore, that the region of early neurogenesis includes ectoderm fated to cease neurogenesis and lie outside of the final placodes. To confirm this, we analyzed the fate of the ectodermal cells in the region where Neurogenin 1 is expressed but which lies outside of the prospective regions of the final placodes (i.e. a region that only expresses Sox3 at early stages).

We labeled a small region of ectoderm dorsocaudal to the prospective region of the petrosal placode with Dil at stage $14^{-}$ or 14 but within the region of early neurogenesis as identified in the experiments above (Fig. 2A compare with Sox3 expression in Fig. 1B). These embryos were allowed to develop for $22 \mathrm{~h}$ until stage 19 (Fig. 2B compare with Sox3 expression in Fig 1E), serially sectioned, photographed under a fluorescent microscope, and immunostained with an antibody against neuron specific class III $\beta$-tubulin (TuJ1). The Dil labeled ectoderm clearly contributed neurons to the cranial ganglia (to the petrosal ganglion in most cases) although no labeled cells were seen in the final placodes (easily identifiable as the source of later TuJ1-positive neuroblasts) (Fig. 2C and D). On the other hand, cells labeled within prospective regions of the final placodes (Fig. 2E) formed an organized stream extending from the surface ectoderm toward the cranial ganglion (Fig. 2E-H). Thus, the ectoderm lying between the final placodes only appears to undergo neurogenesis at early stages. In order to confirm the transient nature of this neuronal contribution, we labeled surface ectoderm of stage 14/14 embryos with Dil in order to determine which regions contributed cells to the ganglia, as described above. We then labeled the same regions with DiO $15 \mathrm{~h}$ after Dil labeling, and then reincubated the 
embryos for $7 \mathrm{~h}$. When such labeling was carried out within the region of the final placodes, inward migration of both Dil- and DiO-labeled neuroblasts was observed (Fig. 2N-Q) demonstrating that this ectoderm contributed neurons to the ganglion at later stages. However, when the region labeled was between the final placodes, we found Dil-positive neurons within the petrosal ganglion while all DiO-labeled cells remained in the surface ectoderm (Fig. 2l-M). Thus, this region of ectoderm is neurogenic during the first $15 \mathrm{~h}$ of the labeling period but is no longer neurogenic during the final $7 \mathrm{~h}$.

We next constructed a fate map of the early post-otic pharyngeal ectoderm by labeling various regions of the pharyngeal ectoderm with Dil. The summary fate map, derived from more than 50 independent injections, is shown in Fig. $2 R$. The region of origin of the cranial ganglia (red+yellow) coincides with the Sox3-positive domain (blue) and includes the ectoderm fated to lie between petrosal and nodose placodes (yellow). These results led us to conclude that the appearance of the final placodes is preceded by the presence of a large continuous placode, which contributes early neurons to the ganglia and is marked by Sox3 expression.

\section{Response of ectoderm to ectopic Neurogenin 2}

The data above suggest that the early large domains of Sox3 expressing ectoderm undergo neurogenesis even though most cells of the cranial ganglia arise from the later, more restricted regions of Sox3 expression (the final placodes). We therefore set out to test the ability of ectoderm within and outside of the
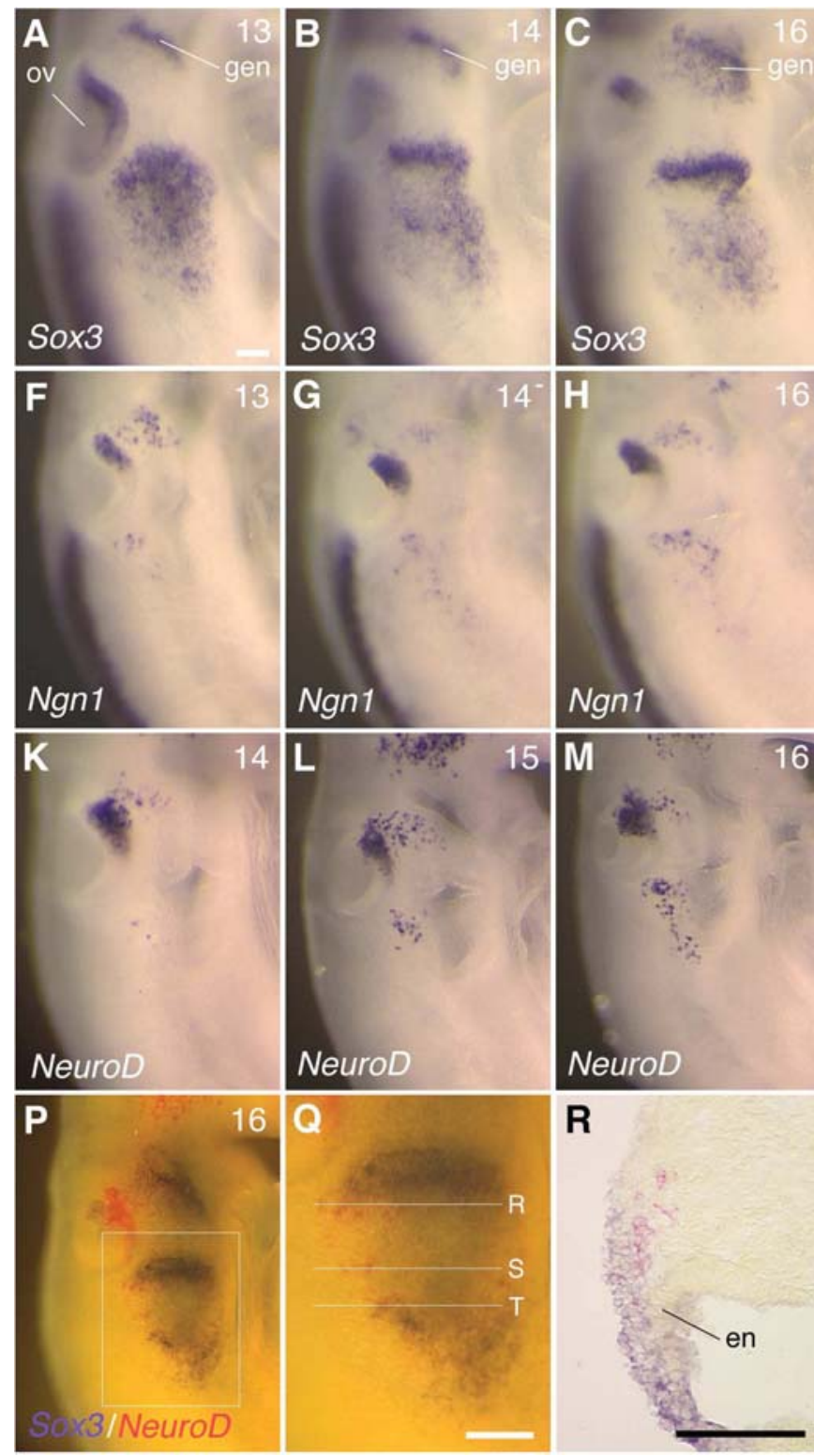

$\mathbf{R}$

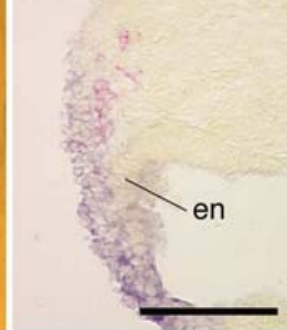

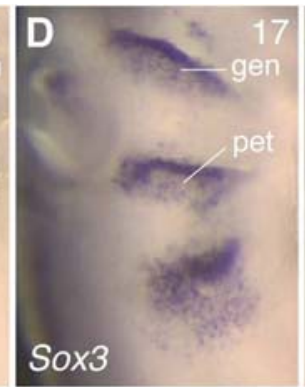
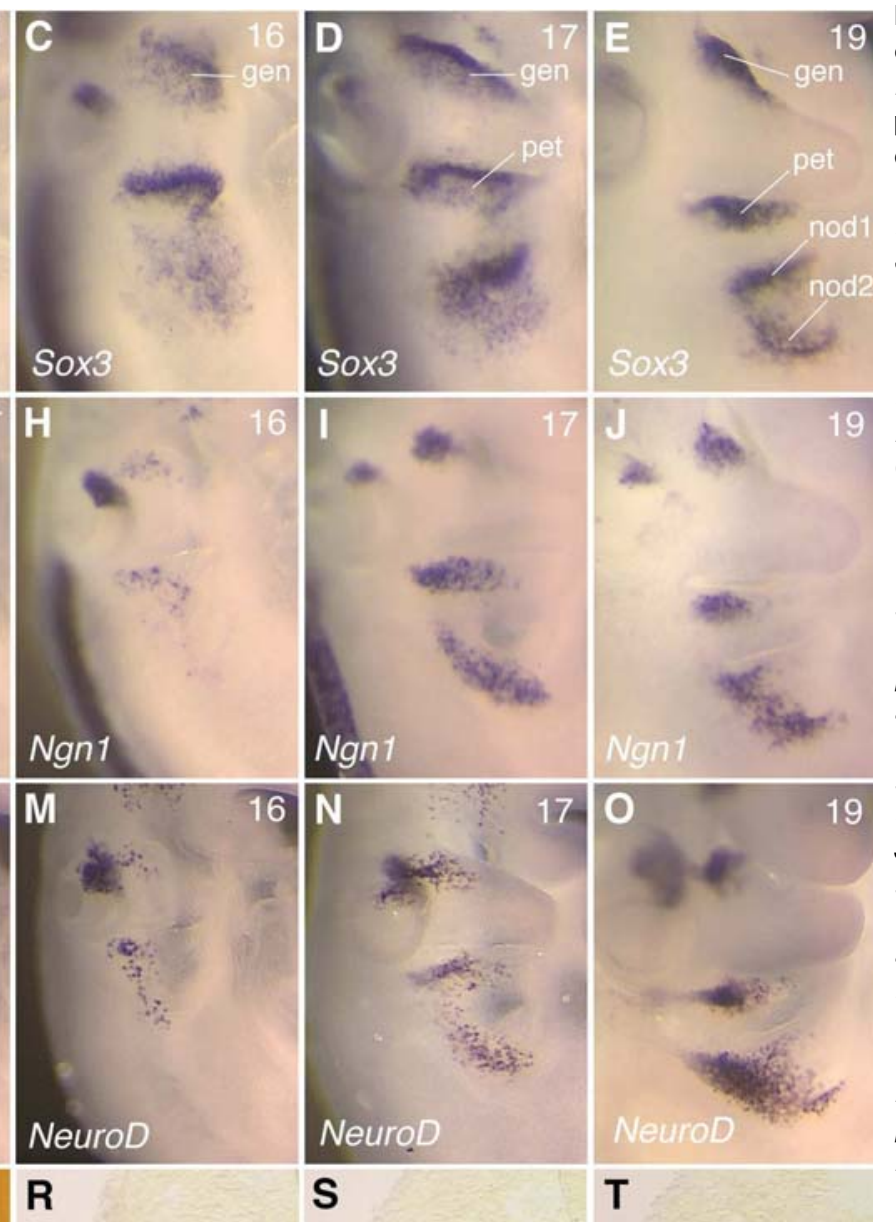

S

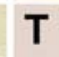

Fig. 1. Dynamic progression of the expression of Sox3, Ngn 1 and NeuroD in the post-otic pharyngeal ectoderm. Lateral view of different stages of embryos hybridized with Sox3 (A-E), Ngn1 (FJ) and NeuroD (K-O). Rostral, up; caudal, down; dorsal left; ventral, right. Numbers in the top right corner refer to the developmental stages (Hamburger and Hamilton, 1951).

(A) Expression of Sox 3 at stage 13 marks a large domain located ventrocaudal to the otic vesicle (ov). (B-E) Sox3 expression becomes restricted to the classically-defined epibranchial placodes (petrosal, firstnodose and second nodose) through the gradual loss of the expression in the intervening ectoderm. Expression of Ngn1 (FJ) and NeuroD (K-O). Expression of Ngn1 and NeuroD becomes detectable by stage 13 and 14 , respectively $(\mathbf{F}, \mathbf{K})$. Its region expands caudally along the dorsal edge of Sox3-positive domain $(\mathbf{G}, \mathbf{H}, \mathbf{L}, \mathbf{M})$, before becoming confined to the final placodes $(\mathbf{I}, \mathbf{J}, \mathbf{N}, \mathbf{O})$. It should be noted in comparing of Sox3 expression with neurogenin 1 and neuroD1 expression at equivalent stages of development, that much of the Neurogenin 1 and NeuroD expression is in cell migrating inwards and does not represent co-expression in the ectodermal cells expressing Sox3. $(\mathbf{P}, \mathbf{0})$ Double wholemount in situ hybridization for Sox3 (blue) and NeuroD (red).

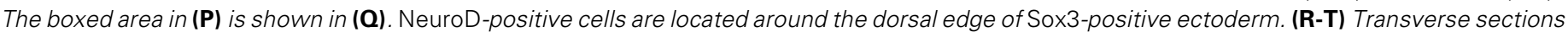
through the levels shown in (Q). NeuroD-positive neuroblasts are produced within Sox3-positive ectoderm regardless of their rostrocaudal levels. gen, geniculate placode; pet, petrosal placode; nod, nodose placode; en, pharyngeal endoderm. Scale bar, $100 \mu \mathrm{m}$. 

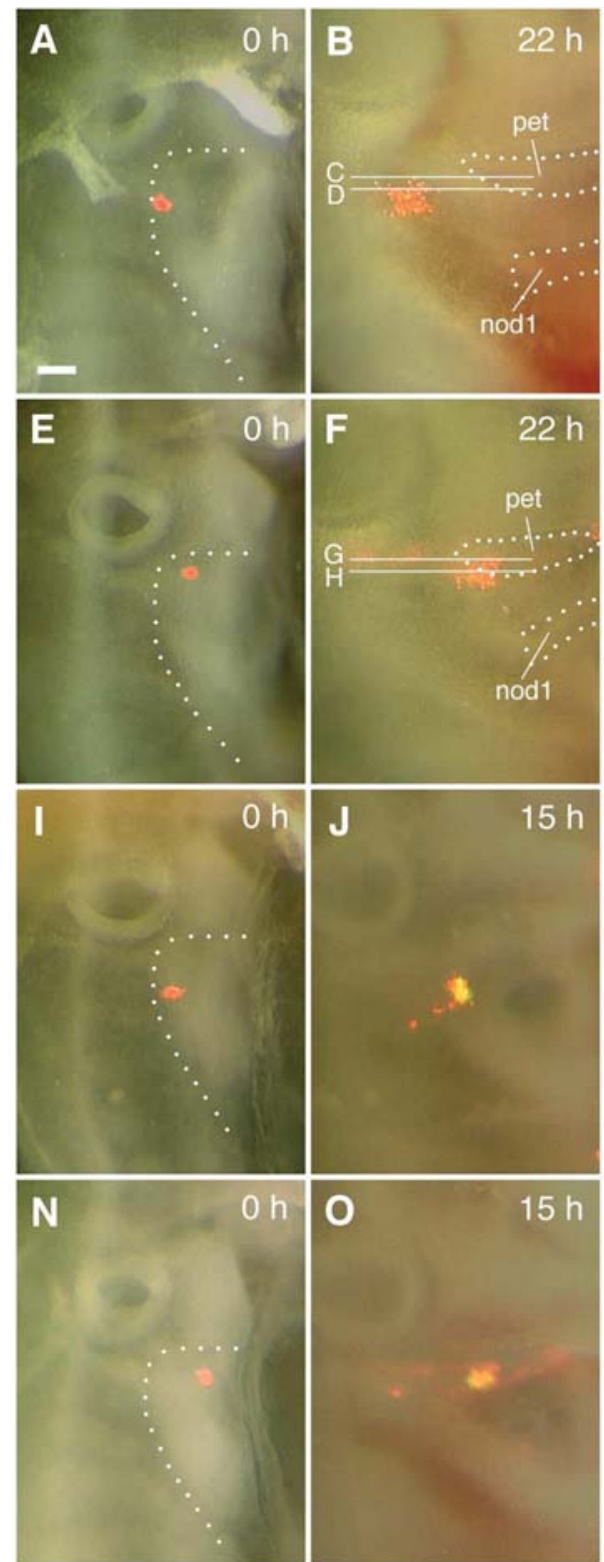
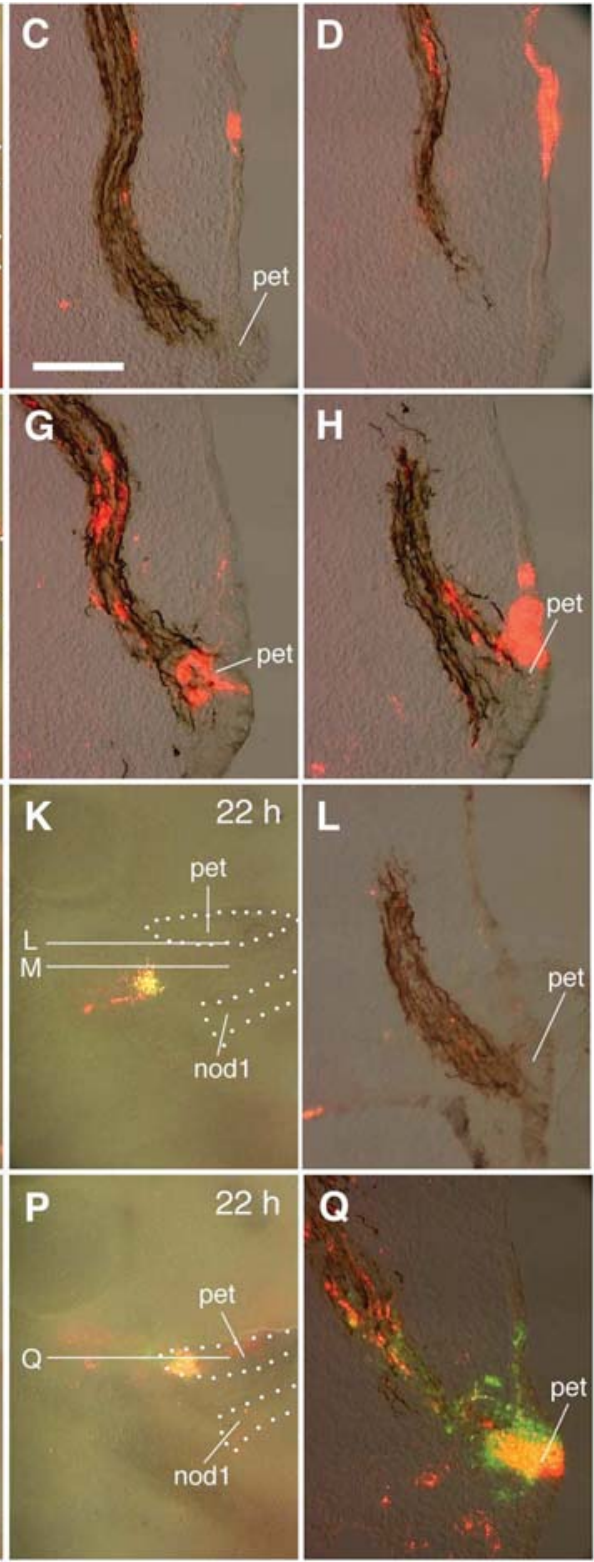

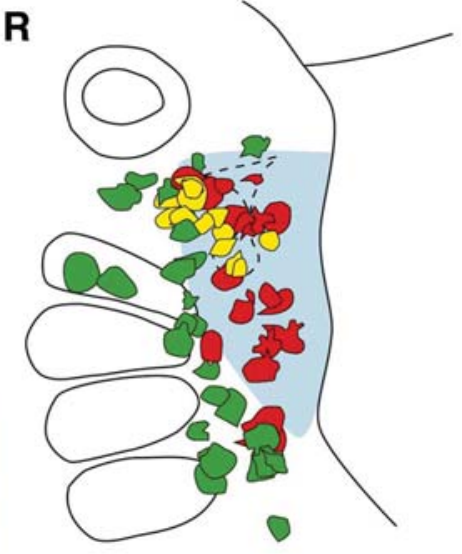

Fig. 2. Shrinking and segmenting areas of placodal neurogenesis.

(A-D) Neurogenesis outside of the prospective regions of the final placodes. Dotted lines indicate Sox3-positive domains, that were carefully determined by comparing with embryos at the same stage stained by wholemount in situ hybridization. (A) A small region of surface ectoderm was labeled with Dil at stage 14114. (B) After $22 \mathrm{~h}$ of incubation the labeled ectoderm contributed outside of the final placode. (C,D) Fluorescence images of transverse frozen sections of the embryo in (B) superimposed upon bright-field images after immunostaining with TuJ1 antibody (brown). Dillabeled cells are seen in the stream of neuronal cells toward the petrosal ganglion despite their absence from the petrosal placodes (pet). (E-H) An embryo in which the prospective region of the petrosal placode was labeled with Dil. Labeled cells produced a stream of neuronal cells extending from the petrosal placode toward the petrosal ganglion. (I-M) Loss of neurogenic activity outside of the final placodes. (I) A small region of ectoderm, similar to that in (A), was labeled with Dil. (J,K) After $15 \mathrm{~h}$ of incubation the same region of surface ectoderm was labeled with DiO, and the embryo was reincubated for $7 \mathrm{~h}$. (L,M) Dil-but no DiO-labeled neurons was seen in the cranial ganglia. (N-O) Prospective region of the petrosal placode was labeled with Dil and then DiO. Dil-positive and DiO-positive neurons are seen in the stream of neurons toward the petrosal ganglion. (R) Summary fate map of the stage 14-/14 embryo. The diagram shows regions that contributed to the final placodes (red), regions that produced neurons but did not contribute to the final placodes (yellow) and regions that produced no neurons (green). Each spot represents a group of cells labeled in a single embryo, $(n=>50)$. Note that the region of origin of cranial ganglia (red+yellow) approximately coincides with Sox3-positive ectoderm (blue). pet, petrosal placode; nod1, first nodose placode; en, pharyngeal endoderm. Scale bar, $100 \mu \mathrm{m}$.

Sox3-expressing regions to respond to neurogenic stimuli. Since Sox3 is a nuclear transcription factor, we first tested the 'transcriptional competence' of the ectoderm, i.e. the ability of neurogenic transcription factors to trigger a response. This was achieved by electroporation of constructs encoding known transcription factors expressed during epibranchial neurogenesis.

Initially we targeted the epibranchial regions of embryos at $\mathrm{HH}$ stage 14 of development, the early stages of neurogenesis, as determined above, and analysed these embryos 16-18 hours later (Fig. 3). We found that NeuroD and NeuroM (using constructs known to function in chick cells (Roztocil et al., 1997, Roztocil et al., 1998)) did not elicit a response in the ectoderm as measured by in situ hybridization for NeuroM, NeuroD or Neurogenin 1 expression (data not shown). Strikingly, Neurogenin2 did elicit a robust and reproducible response $(100 \%, n=55 / 55$, where $n$ is the total number of embryos electroporated). There is strong evidence that 
Neurogenins directly activate expression of NeuroD (Huang et al., 2000, Chae et al., 2004). We found that a similar response was elicited in the ectoderm (Fig. 3, $n=55 / 55$ and Fig.4, $n=36 /$ 36). However, Neurogenin2 failed to trigger delamination and inward migration (Fig. 3Eiii, 4Aiv, 4Avi) or expression of NeuroM and later markers such as $\mathrm{Hu}$ or neurofilament (data not shown). The non-transfected side of the embryo (Fig. 3Biii, 4Aiii), and embryos transfected with a GFP-encoding vector alone $(100 \%$, Fig. $3 A, n=0 / 63)$, did not exhibit an alteration in
NeuroD expression. Although Neurogenin2 elicited strong NeuroD expression in the branchial region of ectoderm, this response was restricted to a ventral cranial region, both trunk $(n=9 / 9)$ and rostral head $(n=11 / 11)$ being unresponsive (Fig. $3 \mathrm{C}, 4 \mathrm{~B})$. We therefore mapped the responsiveness of the ectoderm in relation to the expression domains of Sox3. Embryos were transfected at two stages, $\mathrm{HH} 14$ and $\mathrm{HH} 18 / 19$. These data allowed us to produce maps of responsiveness at these stages (Fig. 3F and Fig. 4C). In general, a broader domain

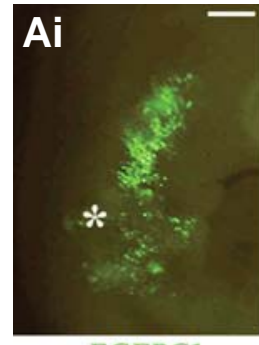

pEGFPC1

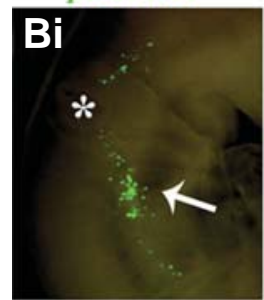

$m N g n 2-G F P$

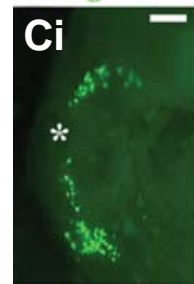

$m N g n 2$ - GFP

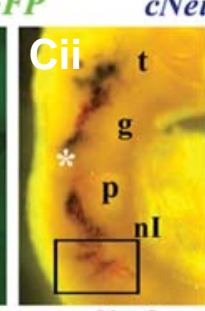

cNeuroD
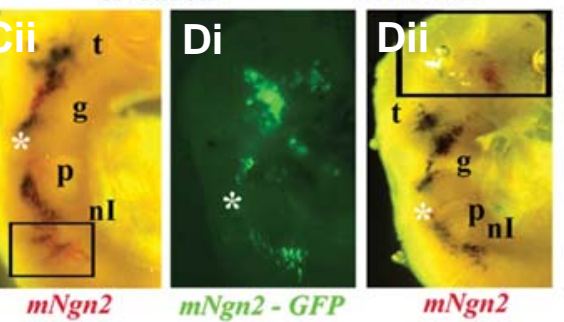
cNeuroD

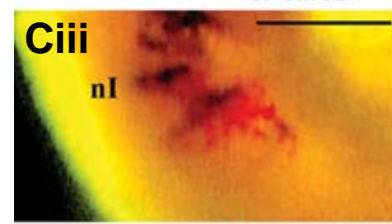

$m N g n 2$ cNeuroD

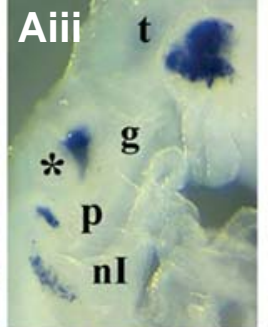

cNeuroD

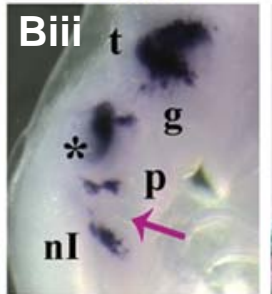

cNeuroD

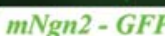

Dif

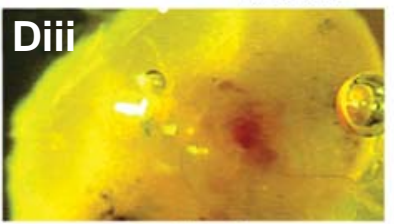

$m N g n 2$

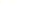
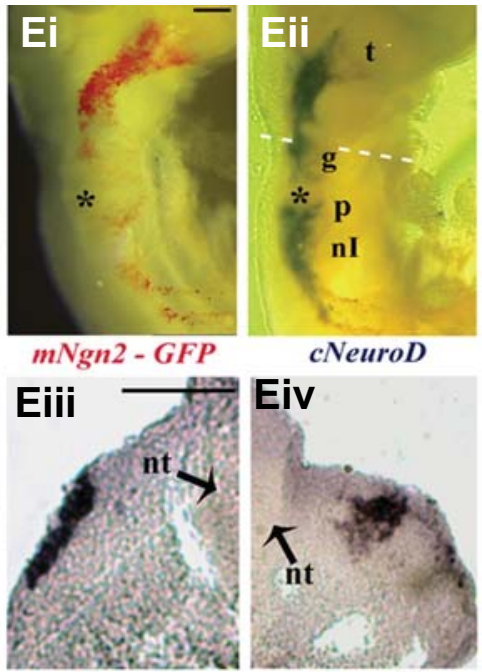

cNeuroD

$\mathbf{F i}$

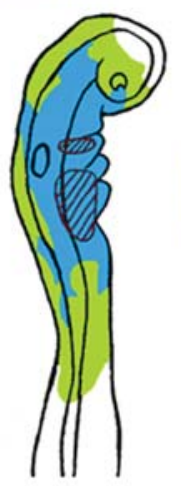

Ec

todermal response to ectopic $m N g n 2$

Responsive regions of the ectoderm

Non-responsive regions of the ectoderm

Expression domains of $\mathrm{cSox} 3$

Fig 3. Effects of over-expression of mNgn2-GFP on the ectoderm at $\mathrm{HH}$ stage 14. Rostral is up and dorsal is to the left. Embryos were electroporated at $\mathrm{HH}$ stage 14 and analysed after 18 hours. mNgn2GFP and pEGFP were detected by green fluorescence. Whole mount single in situ hybridisation was used to detect ectopic mNgn2-GFP in red and $c$ NeuroD in blue. Images of control sides of embryos inverted for ease of comparison. (Ai) Green fluorescence showing transfection of pEGFPC1 in the head and branchial ectoderm. (Aii) In situ hybridisation on the same embryo showing no effect on cNeuroD on the transfected side of the embryo. (Aiii) Endogenous expression of cNeuroD on the non-transfected control side of the embryo. (Bi) Green fluorescence showing transfection of $\mathrm{mNgn2}$ GFP in the region of petrosal and nodose I placodes. (Bii) In situ hybridisation on the same embryo showing activation of cNeuroD. (Biii) Endogenous expression of cNeuroD on control side. Arrows indicate regions of good transfection $(\mathbf{B i}, \mathbf{i i})$ and the equivalent region on the non-transfected side of the embryo in (Biii). (Ci, Di) Green fluorescence showing transfection of $m N g n 2-G F P$ in the region of the geniculate, petrosal and nodose I placodes and also transfection in the head and trunk regions. (Cii, Dii) In situ hybridisation on embryo in (Ci, Di) respectively, showing activation of cNeuroD (blue) in the vicinity of epibranchial placodes, but no activation of $C N e u r o D$ in the head or trunk region where mNgn2-GFP was also transfected (red). (Ciii, Diii) Higher magnification of the boxed regions of embryos in (Cii, Dii) indicating that the cells of the trunk ectoderm posterior to nodose placodes and in more rostral head regions, are not responsive to over-expression of $m$ Ngn2-GFP. (Ei) In situ hybridisation on embryo showing the transfected plasmid detected in red followed by detection of cNeuroD in blue (Eii). (Eiii, iv) Transverse section of embryo in (Eii) at the level of geniculate placodes (white dotted line), showing ectopic expression of $c$ NeuroD on the transfected side and non-transfected control side. cNeuroD is activated only in the surface ectoderm but migration of cells from the ectoderm is not seen (Eiii). On the control side (Eiv) cNeuroD expression is observed in the cells migrating to form the ganglion. (Fi, Fii) Response maps representing data from 55 transfected embryos. Different regions of chick ectoderm show a variation in the response to ectopic $\mathrm{mNgn2}$. Blue colour indicates the regions of ectoderm where a response (activation of cNeuroD) to over-expression of mNgn2-GFP was obtained. The green domains represent regions that were transfected but were unresponsive. The red hatched domains represent the expression pattern of cSox3 at respective stages. (Fii) Responsive and non-responsive regions of the ectoderm as analysed at $\mathrm{HH}$ stage 16, the final stage of analysis when effects of mNgn2-GFP over-expression on cNeuroD were observed. (Fi) Response map of chick ectoderm at HH stage 14 when transfection of mNgn2-GFP was carried out. This map is an estimate of the position of transfected cells at the time of transfection, extrapolated from the regions assayed at the end of the experiment as shown in (Fii). It is evident from both the maps that the responsive regions of ectoderm are much wider than the cSox3 expression domains. Asterisk, otic vesicle; $t$, trigeminal placode; g, geniculate placode; p, petrosal placode; $n l$, nodosel placode; $n t$, neural tube. Scale bars represent approximately $100 \mu \mathrm{m}$. 
of ectoderm was responsive at earlier stages. However, responsiveness was always restricted to regions rostral to the level of the fifth-sixth somites. Dorsal regions were also less responsive, such that at $\mathrm{HH} 14$ most of the branchial region responded except the most dorsal part from about the level of the dorsal part of the otic vesicle. By $\mathrm{HH} 18 / 19$, the non-responsive region had expanded to include the ectoderm dorsal to, and level with, the otic vesicle (Fig $4 \mathrm{Ci}, \mathrm{n}=49$, of which $36 / 36$ in the region shown in blue colour on the map were responsive and 13/13 outside this region (green colour) were negative). Anterior to the branchial clefts, responsiveness was much more restricted only to the ventral most regions including just ventral to the eye and the prospective nasal placode where Sox3 is normally expressed in epithelium fated to form nasal sensory epithelium.

In these experiments we were interested in any correlation between the £regions of responsiveness to Neurogenin 2 and the domains of Sox3 expression. Although there was a significant overlap between these domains, no clear correlation was observed. Most notably, restriction of Sox3 expression to very small placodal regions at $\mathrm{HH}$ stage $18 / 19$ was not reflected in a loss of responsiveness to Neurogenin 2 in the surrounding ectoderm'(Fig. 4). Hence, Sox3 expression does not seem to be required for the competence of ectoderm to express NeuroD in response to ectopic Neurogenin 2.

\section{Response of ectoderm to activated BMP receptor}

Since the response of ectoderm to Neurogenin2 overexpression was limited to activation of NeuroD, we set out to induce a more comprehensive response. To this end, we built on an earlier study in which it was shown that endoderm-derived BMP signals can initiate neurogenesis in and around the normal regions of epibranchial placodes"(Begbie et al., 1999). We made use of a constitutively active receptor for BMP (caBMPR1b (Timmer et al., 2002)). Transfection of ectoderm with this construct allowed us to analyse the effects of activating a BMP pathway in the ectoderm.

As in the experiments using Neurogenin 2, embryos were electroporated at $\mathrm{HH}$ stage $14(\mathrm{n}=39)$ and $\mathrm{HH}$ stage 18/19 $(n=6)$. However, since the caBMPR1b construct did not carry a GFP-tag,
Fig. 4. Effects of overexpression of $\mathbf{m N g n} 2$ GFP on the ectoderm at $\mathrm{HH}$ stage $18 / 19$. Rostral is up and dorsal is to the left. Embryos were electroporated at $\mathrm{HH}$ stage 18/19and analysed after 18 hours. Ectopic mNgn2-GFP was detected by green fluorescence and expression of cNeuroD was detected in blue by whole mount single in situ hybridisation. Images of control sides of embryos inverted for ease of comparison. (Ai) Green fluorescence showing transfection of mNeurogenin2 in the branchial ectoderm and (Aii) activation of $c$ NeuroD in the transfected region. (Aiii) expression of $c$ NeuroD on the non-transfected control side of the same embryo. Dashed white lines in (Aii and Aiii) indicate the level at which the transverse sections were taken. (Aiv-vii) Transverse sections of embryo at the level of geniculate and petrosal placodes, showing ectopic expression of cNeuroD on the transfected side (Aiv, vi), although migration of cells from the ectoderm is not seen. On the control side (Av, vii) cNeuroD expression is observed in the cells migrating to form the ganglion. (Bi) Green fluorescence of embryo in (A) showing transfection of $m$ Ngn2-GFP in the head region. (Bii) Embryo in (Bi) processed through in situ hybridisation showing no ectopic activation of $c$ NeuroD in the transfected region. (Ci, ii) Response maps representing data from 36 transfected embryos. Blue colour indicates the regions of ectoderm where a response (activation of cNeuroD) to over-expression of mNgn2-GFP was obtained. The green domains represent the nonresponsive regions of the ectoderm. The red hatched domains in (Ci) represent the expression pattern of cSox3 at respective stages. (Cii) Responsive and non-responsive regions of the ectoderm as analysed at HH stage 22, the final stage of analysis when effects of mNgn2-GFP over-expression on $c N e u r o D$ were observed. The expression of cSox 3 is lost at this stage and therefore, the expression domains are not represented. (Ci) Response map of chick ectoderm at HH stage 19 when transfection of mNgn2-GFP was carried out. This map is an estimate of the position of transfected cells at the time of transfection, extrapolated from the regions assayed at the end of the experiment as shown in (Cii). It is evident from both the maps that the responsive regions of ectoderm are much wider than the cSox3 expression domains. Asterisk, otic vesicle; $t$, trigeminal placode; $g$, geniculate placode; $p$, petrosal placode; $n \mathrm{l}$, nodose I placode. Scale bars represent approximately $100 \mu \mathrm{m}$. 
co-transfection of the pEGFP-C1 vector was carried out to identify the transfected region.

As anticipated, overexpression of the caBMPR $1 \mathrm{~b}$ elicited expression of NeuroD, (Fig. 5) and these cells also delaminated from the epithelium and migrated inwards (Fig. 5Div).

Again, responsiveness of the ectoderm was restricted to the ventrocaudal ectoderm, with the broadest response in the branchial region. However, the responsiveness to caBMPR did not match the map we produced for the Neurogenin2 response (Fig. $5 E)$. In particular, responsiveness was lost at later stages $(\mathrm{HH} 18$ / 19) (Fig. 5Eii). This pattern of responsiveness coincided more closely with the dynamic domains of Sox3 expression, consistent with a role for Sox3 in this competence to initiate neurogenesis when BMP signaling was activated. These data are consistent with the recent report of Holzschuh et al. (2005), who showed in zebrafish that bmp soaked beads could induce ectopic neurogenesis, but that this was restricted to the branchial ecto- derm (Holzschuh et al., 2005).

\section{Dominant-negative Sox3 inhibits neurogenesis}

Although the analyses above suggested a correlation between Sox3 expression and response of the ectoderm to Bmp signalling, more direct evidence for a role for Sox 3 in this response was needed. In order to investigate directly the requirement of Sox3 expression for neurogenesis in the surface ectoderm, we carried out experiments designed to block Sox3 function. Byland et al. (2004) have demonstrated that Sox3 acts as a transcriptional activator in its role in CNS neurogenesis. We made use of their Sox3-engrailed repressor construct to interfere with Sox3 function in the epibranchial ectoderm (Fig 6). In these experiments we targeted the endogenous domains of Sox3 expression for electroporation by depositing the DNA into the epibranchial clefts, again co-transfecting with pEGFP-C1. This treatment resulted in a striking inhibition of neurogenesis on the treated sides of the
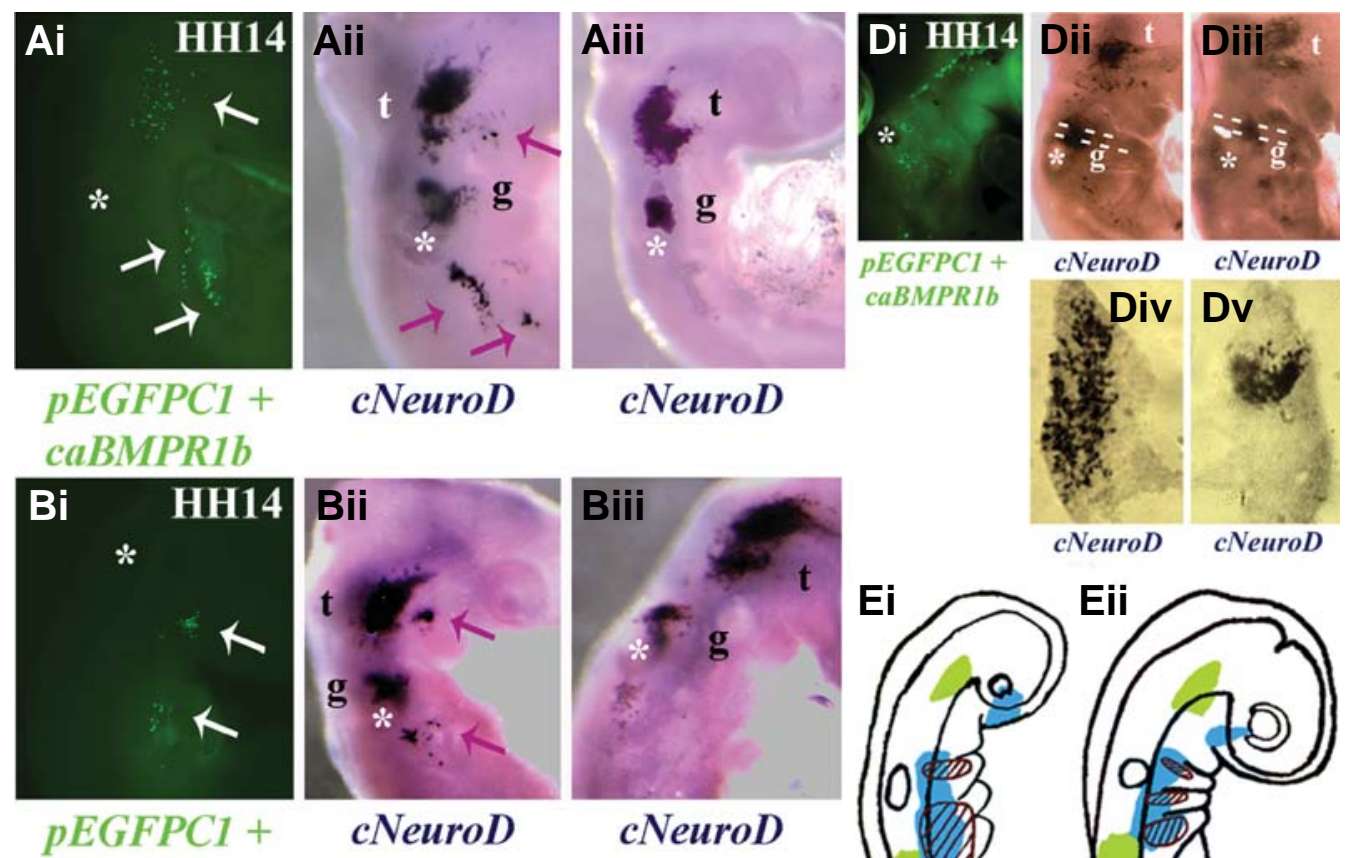
caBMPRIb
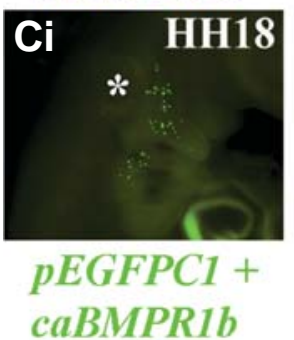

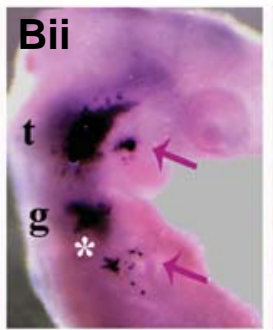

cNeuroD

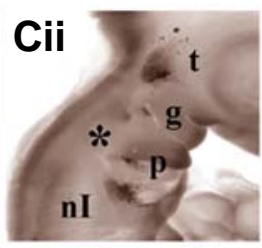

cNeuroD

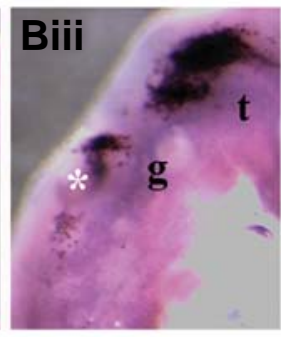

cNeuroD

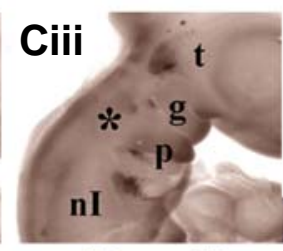

cNeuroD

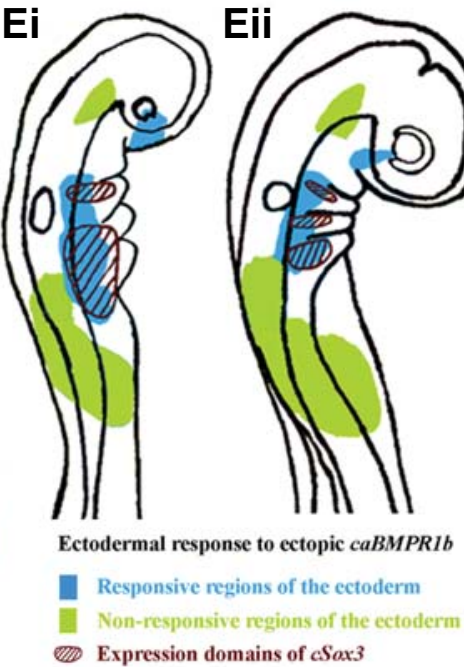

Fig. 5. Effects of caBMPR1b overexpression on the ectoderm at $\mathrm{HH}$ stage 14 and $\mathbf{H H}$ stage 18/19. Embryos were electroporated at $\mathrm{HH}$ stage 14 (A,B,D) or HH stage 18/19 (C) and analysed after 18 hours. Rostral is up and dorsal is to the left. HH stage of transfection in top right corner. Ectopic activation of $c$ NeuroD in response to caBMPR1b over-expression was detected in blue by whole mount single in situ hybridisation. Images of control sides of embryos inverted for ease of comparison. (Ai, Bi, Di) Green fluorescence of GFP showing transfection in the epibranchial region of embryo. (Aii, Bii, Dii) Activation of cNeuroD observed in the transfected regions of the embryos in (Ai, Bi, Di), respectively. (Aiii, Biii, Diii) Non-transfected control side of the embryos in (Ai, Bi, Di), respectively, showing normal endogenous expression of cNeuroD. (Div) Transverse section of embryo in (Di) at the level of geniculate placodes (Dashed white line in Dii, Diii), showing ectopic expression of cNeuroD on the transfected side. Cells expressing ectopic cNeuroD migrated inwards. (Dv) Transverse section of embryo at the level of geniculate placode, showing normal endogenous expression of cNeuroD on the non-transfected control side of the embryo. (Ei, Eii) Response maps representing data collated from 39 transfected embryos. Blue colour indicates the regions of ectoderm where a response (activation of $c$ NeuroD) to over-expression of pMiWIII-caBMPR1b was obtained. The green domains represent the non-responsive regions of the ectoderm. The red hatched domains represent the expression pattern of cSox3 at respective stages. (Eii) Responsive and non-responsive regions of the ectoderm as analysed at HH stage 16, the final stage of analysis when effects of pMiWIII-caBMPR1b over-expression on cNeuroD were observed. (Ei) Response map of chick ectoderm at HH stage 14 when transfection of pMiWIII-caBMPR1b was carried out. This map is an estimate of the position of transfected cells at the time of transfection, extrapolated from the regions assayed at the end of the experiment as shown in (Eii). Arrows indicate regions of transfection. (Ci,ii) No change in expression of cNeuroD was seen upon co-transfection of pEGFPC1 and pMiWIIIcaBMPR1b at HH stage 18/19. (Ciii) Non-transfected control side of the embryo in (Ci) showing normal endogenous expression of cNeuroD. Asterisk, otic vesicle; $t$, trigeminal placode; g, geniculate placode; $p$, petrosal placode; $n$ l, nodose I placode. Scale bars represent approximately 100 um. 
Fig. 6. Effects of over-expression of cSox3-HMG-EnR on the epibranchial placodes at $\mathrm{HH}$ stage 13. Embryos were electroporated at HH stage 13 and analysed at HH stage 15. Ectopic expression of cSox3-HMG-EnR was detected by green fluorescence of co-transfected pEGFPC1 and the endogenous expression of $c$ NeuroD was detected in blue by single in situ hybridisation. Images of
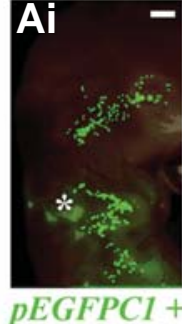
cSox3$-H M G-E n R$ control sides of embryos inverted for ease of comparison. (Ai) Green fluorescence showing co-transfection of pEGFPC1 and cSox3-HMG-EnR (Rostral is up and dorsal is to the left.). (Aii) Embryo in (Ai) showing loss of expression of cNeuroD on the transfected side (dotted lines in (Aii) and (Aiii)). (Aiii) $c$ NeuroD expression on the non-transfected control side of the embryo. (Aiv-vii) Transverse sections of the embryo in (Ai-iii) at the level of geniculate (Aiv), petrosal (Av) and nodose I (Avi, vii) placodes showing loss of cNeuroD on the transfected side of the embryo. Expression of $c$ NeuroD and cell migration are not affected on the non-transfected control side of the embryo (right hand side). (B,C) Embryo cotransfected with cSox3-HMG-EnR and pEGFPC1. Transverse sections at the level of geniculate (Bi), petrosal(Bii) and nodose I (Biii) placodes, showing loss of cell migration on the transfected side of the embryo. (Ci-iii) Embryo transfected with pEGFPC1 alone. Transverse sections at the level of geniculate (Ci), petrosal (Cii) and nodose I (Ciii) placodes, showing normal cell migration on the transfected side of the embryo. nt, neural tube; not, notochord; g, geniculate; $p$, petrosal; nl, nodose l; ph, pharynx. Scale bars represent approximately $100 \mu \mathrm{m}$.
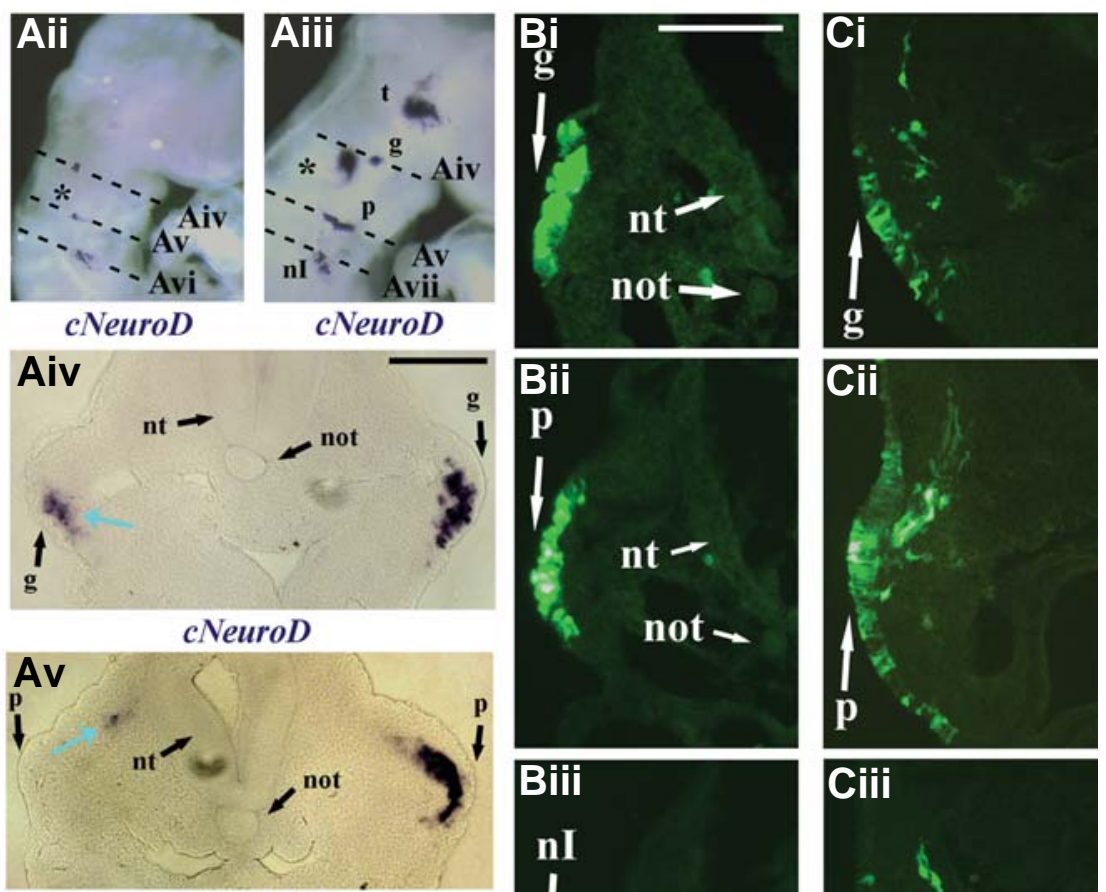

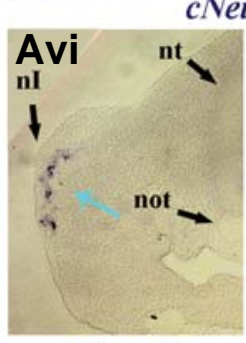

cNeuroD

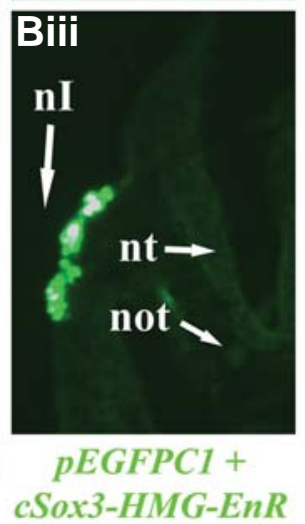

embryos (Fig 6). Not only was there a pronounced decrease in the size of the domain expressing NeuroD and the level of that expression, but there was also very little evidence of the transfected cell migrating inwards (Fig. 6B).

These data are consistent with our recent analysis in zebrafish where knock down of sox 3 by morpholino injection resulted in loss of neurogenesis as marked by expression of either neurogenin or neuroD (unpublished observations).

\section{Discussion}

\section{Sox3 defines dynamically changing areas of neurogenesis}

Our results provide evidence that the epibranchial placodes are dynamically shrinking and segmenting structures. Using Neurogenin 1 and NeuroD as markers for placodal neurogenesis, we have shown the transition of neurogenic regions from an initial longitudinal strip to the final separate patches. The shrinkage of the neurogenic regions was further confirmed by fate mapping study, which identified a region of ectoderm that contributes neurons to cranial ganglia but is fated to lie outside of the final placodes. Although very few cells normally undergo neurogenesis from the regions outside of the classically defined placodes, the activation of NeuroD by ectopic Neurogenin 2 or caBmpR shows that many cells in these regions are actually capable of undergoing this response. However, we cannot be certain whether these cells could generate a full neurogenic response. Together these data suggest the presence of a large continuous domain of neurogenic ectoderm that precedes the appearance of the smaller and segmentally-organized final placodes.

Placodes were originally identified as discrete regions of thickened ectoderm (von Kupffer, 1891). Therefore, neurogenic placodes represent these structures within which sensory neurons or sensory epithelia develop. In some recent studies, the epibranchial placodes are characterized as regions of ectoderm where markers for neurogenesis are expressed (Begbie et al., 1999, Schlosser and Northcutt, 2000, Andermann et al., 2002). However, this criterion appears to be misleading because the region of ectoderm expressing bHLH genes is much smaller than the Sox3-positive thickened ectoderm capable of responding to neurogenic signals. The domain in which neurogenesis actually occurs is likely to be a reflection of the domain capable of neurogenesis and its proximity to the branchial endoderm where Bmp signals arise. Thus, we propose that the epibranchial placode is a pool of uncommitted precursor cells capable of undergoing neuronal differentiation not only the cells that actually undergo neurogenesis.

\section{Variation in the neurogenic response of the ectoderm}

A fundamental and yet poorly understood question of develop- 
mental biology is the basis for competence of cells to undergo neurogenesis in response to appropriate signals. This question has proven extremely difficult to address with respect to the CNS due to the very early stage at which these decisions occur. We have shown that there is spatial and temporal variation in the response of the surface ectoderm to neurogenic stimuli. The ability of ectoderm to undergo neurogenesis in response to activation of BMPR at the cell surface coincides closely to regions of ectoderm that express Sox3 and it seems that Sox3 function may play a direct role in that competence. However, we have found that ectoderm outside of the Sox3-expressing region has the ability to respond to a more direct component of the subsequent neurogenic machinery, the activation of NeuroD expression by Neurogenin 2. The fact that even activation of NeuroD did not result in other aspects of neurogenesis suggests that the ectoderm requires additional factors to be activated that would act in concert with NeuroD to elicit a full neurogenic response. Unfortunately, we have not been able to directly test if it is the expression of Sox3 alone that provides this competence, since overexpression of Sox 3 would also block the progress of cells into neurogenesis. However, our recent study in zebrafish showed that Sox3 was necessary for expression of both neurogenin and phox2a in the placodes, supporting a role for Sox3 in the ability of ectodermal cells to adopt a neurogenic fate (Dee et al., 2008). The maps we have produced and the assays of BMPR and Neurogenin 2 responsiveness, now provide a direct way to access the factors that provide the competence of ectoderm to make neurons.

These regions of competence bear a remarkable similarity to the map produced by Vendrell et al., 2000) describing the ability of ectoderm to form an otic placode in response to ectopically expressed fgf3 (Vendrell et al., 2000). These authors describe competence to be restricted to the dorsal ectoderm adjacent to the hindbrain and the spinal cord as far caudal as the sixth somite, and to be restricted to the cranial ectoderm posterior to the developing eye. This responsive region is very similar to our observation that responsiveness to Neurogenin 2 is restricted to the ventral ectoderm as far caudal as the level of the fifth-sixth somite. Since ours and Vendrell's data identify ventral and dorsal domain respectively with the same caudal limit, this is consistent with a model in which the ectoderm is composed of domains of gene expression which determine the response the ectoderm to various signals. Indeed, Shimada et al., 2003) have found that the competence of ectoderm to express delta-crystallin in response to L-maf is restricted to a domain around the eye, particularly ventral to the eye where we have found that the tissue is also responsive in our study (Shimada et al., 2003). Co transfection of Sox2 expanded this responsiveness suggesting that it was Sox2 expression that provided an essential cofactor for this function of L-Maf and that restricted expression of endogenous Sox2 explained the difference in competence. This restriction of competence for different structures near the eye or more caudally is consistent with our earlier observation that the morphology of ectodermal thickenings produced when Sox3 is over-expressed is more lens-like in rostral ectoderm and epibranchial placode-like in more caudal ectoderm (Abu-Elmagd et al., 2001). What genes might underlie the competence of ventral ectoderm to respond to neurogenic signals? Six 4 is expressed in ventral cranial ectoderm in such a way as to be a potential cofactor for activation of NeuroD by Neurogenin 2. Six4 remains broadly expressed in the branchial arches at later stages (Esteve and Bovolenta, 1999), when we have shown the ectoderm to remain responsive to Neurogenin 2 but not to the caBMPR. Expression of Pax2 however, becomes restricted in a manner similar to Sox3. Since, Pax6 has been shown to be required for Neurogenin 2 expression in the CNS (Scardigli et al., 2003), Pax2 might be necessary for Neurogenin 2 expression in response to BMP in the epibranchial placodes and hence might be a prerequisite for Bmp induced neurogenesis (Baker and Bronner-Fraser, 2000). If both Sox3 and Pax2 are required, might these act together? This is certainly a reasonable hypothesis since Pax6 and SoxB1 genes have been shown to act in concert upon lens specific genes in the cells of the lens placode (Kamachi et al., 2001).

Alternatively, the lack of response to BMP signaling or ectopic Neurogenin 2 could reflect the presence of repressive signals. Recent studies on the expression of genes marking the preplacodal domain which precedes the formation of all placodes, implicated Wnt signaling in such a role (Litsiou et al., 2005). Not only do the expression domain of Wnt8c and particularly Wnt 6 (Schubert et al., 2002) match quite closely to the Neurogenin 2 insensitive regions of ectoderm, but ectopic activation of Wnt signaling was shown to inhibit expression of all preplacodal markers analysed (Litsiou et al., 2005). The role of these various factors in the activation of NeuroD expression by Neurogenin 2 remain to be tested.

\section{Multiple inductive events leading to placodal neuronal differ- entiation}

Our results suggest that the epibranchial placodes retain a potential to undergo epidermal differentiation even after the onset of neurogenesis. In this sense, the placodes contrast with the neural plate, which is irreversibly committed to the nervous system prior to the onset of neurogenesis (Kishi et al., 2000). Such a flexibility of the epibranchial placodes might relate to the fact that they eventually disappear after undergoing active neurogenesis. The placodes cease their neurogenesis by day 5 of development (D'Amico-Martel and Noden, 1983) as soon as the pharyngeal clefts disappear. It is therefore possible that the endoderm is required for maintenance of the placodes and continuance of their neurogenesis. Indeed, the pharyngeal pouch endoderm or BMP-7 is capable of inducing placodal neurogenesis from the surface ectoderm in vitro (Begbie et al., 1999).

However, initial formation of the epibranchial placodes appears to be independent of pharyngeal pouches because Sox3positive ectoderm in the early post-otic region is more extensive than the region of pharyngeal pouch contact. Recent experiments in zebrafish, similarly show that endoderm is required for later neurogenesis (Holzschuh et al., 2005), but not for initial placode formation (Nechiporuk et al., 2005). In culture experiments in vitro, only cranial ectoderm was responsive to the endodermal signal (Begbie et al., 1999). In contrast, an earlier study demonstrated that ectoderm from the trunk can be induced to form neurons when transplanted to the epibranchial region in vivo (Vogel and Davies, 1993). These data suggest that signals from tissues other then the endoderm induce epibranchial placodes and confer the ectoderm with a competence to respond to the endodermal signal, which probably maintains the placodes and facilitates their neurogenesis. Recent studies from our laboratory and others using zebrafish, implicate the Fgf signaling in these 
earlier events (Nechiporuk et al., 2007, Nikaido et al., 2007, Sun et al., 2007) with the mesoderm as the most likely source (Nechiporuk etal., 2007). A similar role for Fgf signaling has been proposed for induction of the otic placode (Phillips et al., 2001, Maroon etal., 2002, Liu et al., 2003, Wright and Mansour, 2003). Fgfs induce otic specific gene expression only in the preplacodal region (reviewed in Streit 2004) that lies adjacent to the CNS (Martin and Groves, 2006). Although it is not yet certain if the prospective epibranchial domain lies within the preplacodal region in chick, our data suggest that it is also induced from within this region in zebrafish (Sun et al., 2007). Since the epibranchial region develops at the same rostrocaudal level as the otic placode and is largely dependent upon Fgf signaling, it seems likely that both classes of placodes are a response to very similar signals.

In summary, we have demonstrated that the epibranchial placodes are dynamically shrinking and segmenting regions of ectoderm capable of undergoing neurogenesis. We have presented evidence that the expression of Sox3 in these structures is a component of their competence to undergo neurogenesis in response to BMP signaling. The ability of neurogenins to induce the expression of NeuroD does not appear to depend upon Sox3 but is restricted to a domain of ectoderm suggesting other factors such as the Six genes might play a role in this response. In order to elucidate the early inductive events, as well as to determine precise roles of the pharyngeal pouch endoderm, it will be particularly important to understand mechanisms underlying commitment of ectodermal cells to a neural fate. Sox3 provides a valuable tool to investigate this process.

\section{Materials and Methods}

\section{Embryos}

White Leghorn chicken eggs were incubated at $38^{\circ} \mathrm{C}$. Embryos were staged according to Hamburger and Hamilton (1951) (Hamburger and Hamilton, 1951).

\section{Whole-mount in situ hybridization}

cDNAs for Sox3 (Uwanogho etal., 1995), Neurogenin 1 and Neurogenin 2 (gift from Dr. David Anderson) and NeuroD (gift from Dr. Marc Ballivet) were used to make antisense riboprobes. Neurogenin 2 was used to make sense probes to detect the over-expressed plasmid. Whole-mount in situ hybridization was performed as described previously (Cheung et al., 2000). For double whole-mount in situ hybridization, the method described by Abu-Elmagd et al. (2001) was used. The fluorescein-labeled Neurogenin 2 probe and digoxigenin-labeled NeuroD probe were hybridized simultaneously. Expression of Neurogenin 2 was detected first by using Fast Red TR/Naphthol AS-MX (Sigma), followed by the detection of NeuroD using NBT/BCIP.

\section{Fate mapping}

Dil (1,1-dioctadecyl-3,3,3',3'-tetramethylindo-carbocyanineperchloride; Molecular Probes, Eugene, OR; $0.3 \%$ in dimethylformamide) was applied on the surface ectoderm of stage 14 or 14 (21 or 22 somites stage) embryos using a fine glass microcapillary. Photographs were taken immediately with a CCD camera (DC-330, DAGE-MIT Inc., Michigan City, IN) under a fluorescent microscope. Positions and sizes of the labeled ectoderm were carefully determined in relation to somites, pharyngeal pouches and otic vesicle. The embryos were incubated for $22 \mathrm{~h}$, but some were labeled with $\mathrm{DiO}$ (3,3'-dioctadecyloxacarbocyanineperchlorate; Molecular Probes) at $15 \mathrm{~h}$ then reincubated for $7 \mathrm{~h}$. The embryos were fixed with $4 \%$ paraformaldehyde/phosphate buffered saline for 1-2 $\mathrm{h}$, embedded in OCT compound and serially cryosectioned. After being photographed under a fluorescent microscope, sections containing labeled cells were immunostained with monoclonal antibody against neuron specific class III $\beta$-tubulin (TuJ1, Babco, Berkeley, CA, 1:500) to visualize streams of neurons extending from the placodal ectoderm towards the cranial ganglia. Biotinylated anti-mouse IgG (1:200, Vector laboratories, Burlingame, CA) and ABC-kit (Vector laboratories) were used to detect the immunoreactivity.

\section{Electroporation}

Clones for Neurogenin2 fused with pEGFPN1, caBMPR (Timmer, et. al. 2002), cSox3-HMG-EnR (Bylund et. al., 2003) were used for overexpression. mNeurogenin2-pEGFPN1 and pMiWIII-caBMPR1b + pEGFPC1 were transfected at $\mathrm{HH}$ stage 14 and $\mathrm{HH}$ stage $18 / 19$ and analysed at $\mathrm{HH}$ stage 16 and $\mathrm{HH}$ stage $21 / 22$ respectively. CSOX3-HMGEnR was co-transfected with $p E G F P C 1$ at $\mathrm{HH}$ stage 13 and embryos were analysed at $\mathrm{HH}$ stage 15.

\section{Histological analysis}

Embryos were fixed in Bouin's fluid, dehydrated in a graded ethanol series, embedded in wax and sectioned longitudinally at $7 \mu \mathrm{m}$. The sections were mounted on vectabond (Vector laboratories)-coated slides and stained with Mayer's hematoxylin (Sigma) and eosin.

\section{Acknowledgments}

We would like to thank to David J. Anderson (Ngn1 and Ngn2 probe) and Marc Ballivet (NeuroM, and NeuroD clones). Thanks to Jonas Muhr for cSox3-HMG-EnR construct and Lee Niswander for caBMPR1b construct.

\section{References}

ABU-ELMAGD, M., ISHII, Y., CHEUNG, M., REX, M., LE ROUEDEC, D. and SCOTTING, P. (2001). Csox3 expression and neurogenesis in the epibranchial placodes. Dev. Biol. 237: 258-269.

BAKER, C. and BRONNER-FRASER, M. (2000). Establishing neuronal identity in vertebrate neurogenic placodes. Development 127: 3045-3056.

BAKER, C. and BRONNER-FRASER, M. (2001). Vertebrate cranial placodes i. Embryonic induction. Dev. Biol. 232: 1-61.

BALLY-CUIF, L. and HAMMERSCHMIDT, M. (2003). Induction and patterning of neuronal development, and its connection to cell cycle control. Curr Opin Neurobio/13: 16-25

BEGBIE, J., BRUNET, J.-F., RUBENSTEIN, J. and GRAHAM, A. (1999). Induction of the epibranchial placodes. Development 126: 895-902.

CHAE, J.H., STEIN, G.H. and LEE, J.E. (2004). Neurod: The predicted and the surprising. Mol Cel/s 18: 271-88.

CHEUNG, M., ABU-ELMAGD, M., CLEVERS, H. and SCOTTING, P.J. (2000) Roles of sox4 in central nervous system development. Mol. Brain Res. 79: 18091.

D'AMICO-MARTEL, A. and NODEN, D. (1983). Contributions of placodal and neural crest cells to avian cranial peripheral ganglia. Am. J. Anat. 166: 445-468.

DEE, C.T., HIRST, C.S., SHIH, Y.-H., TRIPATHI, V.B., PATIENT, R.K. and SCOTTING, P.J. (2008). Sox3 regulates both neural fate and differentiation in the zebrafish ectoderm. Dev. Biol. 320: 289-301.

ESTEVE, P. and BOVOLENTA, P. (1999). Csix4, a member of the six gene family of transcritpion factors, is expressed during placode and somite development. Mech. Dev. 85: 161-165.

FODE, C., GRADWOHL, G., MORIN, X., DIERICH, A., LEMEUR, M., GORIDIS, C and GUILLEMOT, F. (1998). The bhlh protein neurogenin 2 is a determination factor for epibranchial placode-derived sensory neurons. Neuron 20: 483-494

GRAHAM, A. and BEGBIE, J. (2000). Neurogenic placodes: A common front. Trends in Neurosciences 23: 313-316.

HAMBURGER, V. and HAMILTON, H. (1951). A series of normal stages in the development of the chick embryo. Journal of Morphology 88: 49-92.

HOLZSCHUH, J., WADA, N., WADA, C., SCHAFFER, A., JAVIDAN, Y., TALLAFUSS, 
A., BALLY-CUIF, L. and SCHILLING, T.F. (2005). Requirements for endoderm and bmp signaling in sensory neurogenesis in zebrafish. Development 132: 3731-42.

HUANG, H.P., LIU, M., EL-HODIRI, H.M., CHU, K., JAMRICH, M. and TSAI, M.J. (2000). Regulation of the pancreatic islet-specific gene beta2 (neurod) by neurogenin 3. Mol Cel/ Bio/20: 3292-307.

ISHII, Y., ABU-ELMAGD, M. and SCOTTING, P.J. (2001). Sox3 expression defines a common primordium for the epibranchial placodes in chick. Dev. Biol. 344353.

KAMACHI, Y., UCHIKAWA, M., TANOUCHI, A., SEKIDO, R. and KONDOH, H. (2001). Pax6 and sox2 form a co-DNA-binding partner complex that regulates initiation of lens development. Genes Dev 15: 1272-86.

KISHI, M., MIZUSEKI, K., SASAI, N., YAMAZAKI, H., SHIOTA, K., NAKANISHI, S. and SASAI, Y. (2000). Requirement of sox2-mediated signaling for differentiation of early Xenopus neuroectoderm. Development 127: 791-800.

LE DOUARIN, M.N., FONTAINE-PE'RUS, J. and COULY, G. (1986). Cephalic ectodermal placodes and neurogenesis. Trends in Neuroscience 9: 175-180.

LITSIOU, A., HANSON, S. and STREIT, A. (2005). A balance of fgf, bmp and wnt signalling positions the future placode territory in the head. Development 132 : 4051-62.

LIU, D., CHU, H., MAVES, L., YAN, Y.L., MORCOS, P.A., POSTLETHWAIT, J.H. and WESTERFIELD, M. (2003). Fgf3 and fgf8 dependent and independent transcription factors are required for otic placode specification. Development 130: 2213-24.

MAROON, H., WALSHE, J., MAHMOOD, R., KIEFER, P., DICKSON, C. and MASON, I. (2002). Fgf3 and fgf8 are required together for formation of the otic placode and vesicle. Development 129: 2099-2108.

MARTIN, K. and GROVES, A.K. (2006). Competence of cranial ectoderm to respond to fgf signaling suggests a two-step model of otic placode induction. Development 133: 877-87.

NARAYANAN, C.H. and NARAYANAN, Y. (1980). Neural crest and placodal contributions in the development of the glossopharyngeal-vagal complex in the chick. Anat Rec 196: 71-82.

NECHIPORUK, A., LINBO, T., POSS, K.D. and RAIBLE, D.W. (2007). Specification of epibranchial placodes in zebrafish. Development 134: 611-23.

NECHIPORUK, A., LINBO, T. and RAIBLE, D.W. (2005). Endoderm-derived fgf3 is necessary and sufficient for inducing neurogenesis in the epibranchial placodes in zebrafish. Development 132: 3717-30.

NIKAIDO, M., KAZUNAO, D., TAKASHI, S., MASAHIKO, H., YUTAKA, K. and KYO, Y. (2007). Initial specification of the epibranchial placode in zebrafish embryos depends on the fibroblast growth factor signal. Dev. Dynam. 236: 564-571.

PEREZ, S., REBELO, S. and ANDERSON, D. (1999). Early specification of sensory neuron fate revealed by expression and function of neurogenins in the chick embryo. Development 126: 1715-1728

PHILLIPS, B.T., BOLDING, K. and RILEY, B.B. (2001). Zebrafish fgf3 and fgf8 encode redundant functions required for otic placode induction. Dev. Biol. 235 351-365

SCARDIGLI, R., BAUMER, N., GRUSS, P., GUILLEMOT, F. and LE ROUX, I. (2003). Direct and concentration-dependent regulation of the proneural gene neurogenin2 by pax6. Development 130: 3269-81.

SCHUBERT, F.R., MOOTOOSAMY, R.C., WALTERS, E.H., GRAHAM, A., TUMIOTTO, L., MUNSTERBERG, A.E., LUMSDEN, A. and DIETRICH, S. (2002). Wnt6 marks sites of epithelial transformations in the chick embryo. Mech Dev 114: 143-8.

SHIMADA, N., AYA-MURATA, T., REZA, H.M. and YASUDA, K. (2003). Cooperative action between I-maf and sox2 on delta-crystallin gene expression during chick lens development. Mech Dev120: 455-65.

SUN, S.-K., DEE, C.T., TRIPATHI, V.B., RENGIFO, A., HIRST, C.S. and SCOTTING, P.J. (2007). Epibranchial and otic placodes are induced by a common fgf signal, but their subsequent development is independent. Dev. Biol. 303: 675686.

TIMMER, J.R., WANG, C. and NISWANDER, L. (2002). Bmp signaling patterns the dorsal and intermediate neural tube via regulation of homeobox and helix-loophelix transcription factors. Development 129: 2459-72.

VENDRELL, V., CARNICERO, E., GIRALDEZ, F., ALONSO, M. and SCHIMMANG T. (2000). Induction of inner ear by fgf3. Development 127: 2011-2019.

VOGEL, K. and DAVIES, A. (1993). Heterotopic transplantation of presumptive placodal ectoderm changes the fate of sensory neuron precursors. Develop ment 119: 263-276.

WEBB, J. and NODEN, D. (1993). Ectodermal placodes: Contributions to the development of the vertebrate head. American Zoology 33: 434-447.

WRIGHT, T.J. and MANSOUR, S.L. (2003). Fgf3 and fgf 10 are required for mouse otic placode induction. Development 130: 3379-90.
5 yr ISI Impact Factor $(2008)=3.271$

\section{For all the latest on Pattern Formation research, see our latest Special Issue edited by C.-M. Chuong and M.K. Richardson.}

http://www.ijdb.ehu.es/web/contents.php?vol=53\&issue=5-6

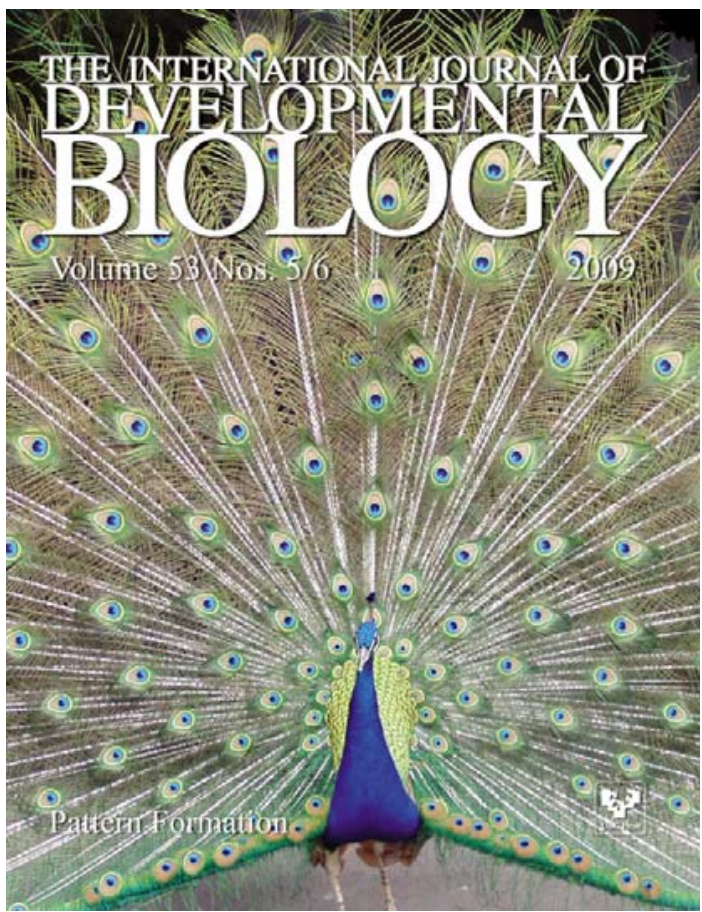




\section{Further Related Reading, published previously in the Int. J. Dev. Biol.}

See our recent Special Issue Fertilization, in honor of David L. Garbers and edited by Paul M. Wassarman and Victor D. Vacquier at: http://www.ijdb.ehu.es/web/contents.php?vol=52\&issue=5-6

Cloning and developmental expression of the soxB2 genes, sox14 and sox21, during Xenopus laevis embryogenesis Doreen D. Cunningham, Zhuo Meng, Bernd Fritzsch and Elena Silva Casey Int. J. Dev. Biol. (2008) 52: 999-1004

Loss of Sox9 function results in defective chondrocyte differentiation of mouse embryonic stem cells in vitro Gunnar Hargus, Ralf Kist, Jan Kramer, Daniela Gerstel, Angela Neitz, Gerd Scherer and Jürgen Rohwedel Int. J. Dev. Biol. (2008) 52: 323-332

The first steps towards hearing: mechanisms of otic placode induction Takahiro Ohyama, Andrew K. Groves and Kareen Martin Int. J. Dev. Biol. (2007) 51: 463-472

Expression and regulation of Xenopus CRMP-4 in the developing nervous system Jacob Souopgui, Tiemo J. Klisch, Tomas Pieler and Kristine A. Henningfeld Int. J. Dev. Biol. (2007) 51: 339-343

Expression of Bmp ligands and receptors in the developing Xenopus retina Jennifer C. Hocking and Sarah McFarlane Int. J. Dev. Biol. (2007) 51: 161-165

A change in response to Bmp signalling precedes ectodermal fate choice Chris T. Dee, Abigail Gibson, Andrea Rengifo, Shun-Kuo Sun, Roger K. Patient and Paul J. Scotting Int. J. Dev. Biol. (2007) 51: 79-84

BMP signalling in craniofacial development Xuguang Nie, keijo Luukko and Paivi Kettunen Int. J. Dev. Biol. (2006) 50: 511-521

Expression of regulatory genes for pancreas development during murine embryonic stem cell differentiation Josué K. Mfopou, Erik Willems, Luc Leyns and Luc Bouwens Int. J. Dev. Biol. (2005) 49: 915-922

Transforming growth factor beta2 promotes the formation of the mouse cochleovestibular ganglion in organ culture Junko Okano, Toshiya Takigawa, Kenji Seki, Shigehiko Suzuki, Kohei Shiota and Makoto Ishibashi Int. J. Dev. Biol. (2005) 49: 23-31

Interplay of Pax6 and SOX2 in lens development as a paradigm of genetic switch mechanisms for cell differentiation

Hisato Kondoh, Masanori Uchikawa and Yusuke Kamachi

Int. J. Dev. Biol. (2004) 48: 819-827

Sox9, a novel pancreatic marker in Xenopus.

Young-Hoon Lee and Jean-Pierre Saint-Jeannet

Int. J. Dev. Biol. (2003) 47: 459-462

Roles of Sox factors in neural determination: conserved signaling in evolution? Y Sasai

Int. J. Dev. Biol. (2001) 45: 321-326

Cell morphology in amphioxus nerve cord may reflect the time course of cell differentiation.

T C Lacalli

Int. J. Dev. Biol. (2000) 44: 903-906

Retinoid signalling acts during the gastrula stages to promote primary neurogenesis.

C Sharpe and K Goldstone

Int. J. Dev. Biol. (2000) 44: 463-470

5 yr ISI Impact Factor $(2008)=3.271$

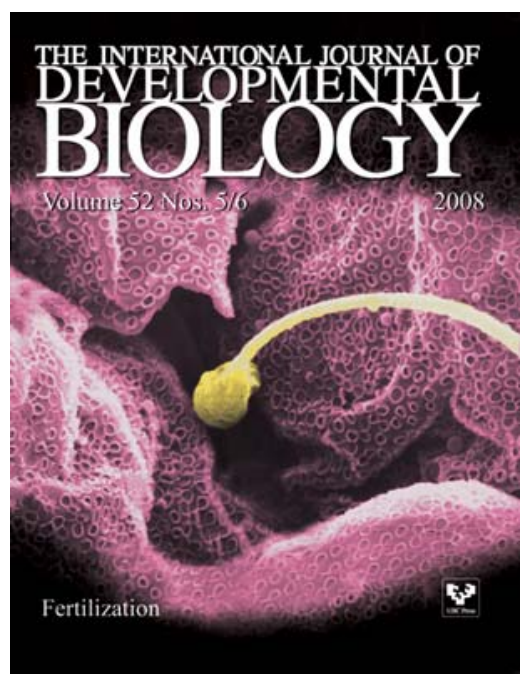

